\title{
Relación entre el polimorfismo rs1414334 C/G del gen HTR2C y tabaquismo en pacientes tratados con antipsicóticos atípicos
}

\author{
Relationship between the rs1414334 C/G \\ polymorphism in the HTR2C gene and smoking in \\ patients treated with atypical antipsychotics
}

\begin{abstract}
José María Rico-Gomis*, Antonio Palazón-Bru**, Irene Triano-García***, Luis Fabián Mahecha-García*, Ana García-Monsalve***, Andrés Navarro-Ruiz***, Berta VillagordoPeñalver*, Alicia Martínez-Hortelano*, Vicente Francisco Gil-Guillén***

* Unidad de Psiquiatría, Hospital General Universitario de Elche, Elche, Alicante, España. ** Departamento de Medicina Clínica, Universidad Miguel Hernández, San Juan de Alicante, Alicante, España. *** Servicio de Farmacia, Hospital General Universitario de Elche, Elche, Alicante, España.
\end{abstract}

\section{Resumen}

En pacientes psiquiátricos, otros autores han encontrado una asociación entre el alelo C del polimorfismo rs1414334 del gen HTR2C y el síndrome metabólico. Ninguno de ellos ha valorado si este alelo se asocia con el consumo de tabaco, por lo que se decidió realizar un estudio en una región española valorando esta cuestión. Estudio observacional transversal de una muestra de 166 pacientes adultos tratados con antipsicóticos atípicos en 20122013. Variable principal: presencia del alelo $\mathrm{C}$ del polimorfismo rs1414334 del gen HTR2C. Variables secundarias: número de añospaquete (número de cigarrillos al día $\mathrm{x}$ número de años fumando $\div 20$ ), edad, sexo, esquizofrenia, años diagnosticados del trastorno, criterios de síndrome metabólico y SCORE. Se construyó un modelo de regresión logística binaria por pasos para determinar asociaciones entre la variable principal y las variables secundarias del estudio y se calculó su área bajo la curva ROC (ABC). Del total de la muestra, 33 pacientes presentaron el alelo $\mathrm{C}$ del polimorfismo analizado (19,9\%). El consumo de tabaco medio fue de 11.6 paquetes-año. El modelo multivariante arrojó los siguientes factores asociados al polimorfismo: mayor consumo tabáquico, ser mujer y no tener obesidad abdominal. El ABC fue de 0,706. Se ha encontrado asociación entre un mayor consumo de tabaco a lo largo de los años y la presencia del alelo $\mathrm{C}$ del polimorfismo rs1414334 del gen HTR2C. Se necesitan trabajos que corroboren nuestros resultados.

Palabras clave: Tabaquismo; Farmacogenética; Alelos; Psiquiatría.

\begin{abstract}
An association has been found between the $\mathrm{C}$ allele of the rs1414334 polymorphism in the HTR2C gene and the metabolic syndrome in psychiatric patients. However, no study has yet evaluated whether this allele is associated with smoking. To assess this issue, therefore, we performed a cross-sectional study with a sample of 166 adult patients treated with atypical antipsychotics in 2012-2013 in a region of Spain. The primary variable was the presence of the C allele of the rs1414334 polymorphism in the HTR2C gene. Secondary variables were the number of pack-years (number of cigarettes per day $\mathrm{x}$ number of smoking years $\div 20$ ), age, gender, schizophrenia, years since diagnosis, metabolic syndrome criteria and SCORE. A stepwise binary logistic regression model was constructed to determine associations between primary and secondary variables and their area under the ROC curve (AUC) was calculated. Of the total sample, 33 patients (19.9\%) had the $\mathrm{C}$ allele of the polymorphism analyzed. Mean cigarette consumption was 11.6 pack-years. The multivariate analysis showed the following factors as associated with the polymorphism: higher cigarette consumption, being a woman, and not having abdominal obesity. The AUC was 0.706. An association was found between increased cigarette consumption over the years and the presence of the $\mathrm{C}$ allele of the rs1414334 polymorphism in the HTR2C gene. Keywords: Smoking; Pharmacogenetics; Alleles; Psychiatry.
\end{abstract}


$\mathrm{E}$ 1 receptor HTR2C tiene diversas funciones, entre ellas se encuentran la regulación del apetito, homeostasis de la glucosa y también se relaciona con el uso de sustancias psicoactivas. Se ha relacionado con patologías como el trastorno ciclotímico, el suicidio o la eyaculación precoz (The Weizmann Institute of Science Crown Human Genome Centre, 2014).

En lo que se refiere a la literatura existente sobre el polimorfismo rs1414334 del gen HTR2C, ésta se centra en la relación de la presencia del alelo $\mathrm{C}$ de este polimorfismo en pacientes psiquiátricos en tratamiento con antipsicóticos y la posible relación con la aparición de síndrome metabólico, valorando tanto cada uno de los componentes de dicho síndrome como el conjunto de las mismos. En los resultados de estos trabajos generalmente se aprecia una asociación a favor entre el alelo $\mathrm{C}$ de este polimorfismo y el síndrome metabólico (Gregoor et al., 2010; Hoekstra et al., 2010; Houston et al., 2012; Klemettilä et al., 2015; Ma et al., 2014; Mulder et al., 2007a; Mulder et al., 2007b; Mulder et al., 2009; Rico-Gomis et al., 2016; Risselada et al., 2012). En otras palabras, estos pacientes presentan un mayor riesgo cardiovascular. Por otra parte, hemos de tener en cuenta que existe otro factor de riesgo fundamental en el desarrollo de la patología cardiovascular que no se contempla en los criterios del síndrome metabólico, el cual es el tabaquismo (Conroy et al., 2003; Wilson et al., 1998). Ninguno de los estudios anteriores ha valorado si los pacientes con este alelo tienen una asociación con el consumo de tabaco (Gregoor et al., 2010; Hoekstra et al., 2010; Houston et al., 2012; Klemettilä et al., 2015; Ma et al., 2014; Mulder et al., 2007a; Mulder et al., 2007b; Mulder et al., 2009; Rico-Gomis et al., 2016; Risselada et al., 2012). Ésta es una cuestión relevante, ya que en modelos animales se ha observado como el receptor HTR2C modula la adicción a la nicotina en ratones y por otra parte se ha determinado que la estimulación de los receptores HTR2C reduce la función de la dopamina a nivel mesolímbico (Guy y Fletcher, 2014), disminuyendo los efectos estimulantes de la nicotina (Fletcher, Lê y Higgins, 2008). Teniendo en cuenta la falta de estudios (Gregoor et al., 2010; Hoekstra et al., 2010; Houston et al., 2012; Klemettilä et al., 2015; Ma et al., 2014; Mulder et al., 2007a; Mulder et al., 2007b; Mulder et al., 2009; Rico-Gomis et al., 2016; Risselada et al., 2012), la posible asociación del tabaquismo con este receptor y que se ha encontrado dependencia al tabaco con otros genes (Barrot, Sánchez, Abellana, Ortega y Gené, 2013; Fletcher et al., 2008; Guy y Fletcher, 2014; Saccone et al., 2007; Verde et al., 2011; Walton, Johnstone, Munafò, Neville y Griffiths, 2001), decidimos realizar un estudio valorando la posible asociación del consumo tabáquico con el alelo C del polimorfismo rs1414334 del gen HTR2C en pacientes psiquiátricos con antipsicóticos atípicos. Los resultados aportarán evidencia científica sobre el consumo de sustancias tóxicas por parte de estos pacientes y servirán para mejorar las directrices clínicas aplicables a patologías duales. Por lo tanto, mejorará el éxito de los tratamientos de cesación tabáquica en esta población (San et al., 2016).

\section{Métodos}

\section{Consideraciones éticas}

Todos los pacientes fueron debidamente informados y rellenaron un formulario de consentimiento informado antes de participar en este estudio. El Comité de Ética e Investigación del Hospital General Universitario de Elche aprobó el estudio el 20 de noviembre de 2012. Todos los procedimientos siguieron las normas de la Declaración de Helsinki revisadas en el 2004.

\section{Población estudiada}

La población estudiada estaba compuesta de pacientes psiquiátricos tratados con antipsicóticos atípicos en el Departamento de Salud 20 (Hospital General Universitario de Elche). Esta Unidad está ubicada en la Comunidad Valenciana, una región Mediterránea en el sureste de España de aproximadamente 5 millones de habitantes. En esta Unidad, la cobertura sanitaria para pacientes psiquiátricos es universal y gratuita.

\section{Diseño del estudio y participantes}

Estudio observacional transversal de una muestra de todos los pacientes adultos que acudieron a los servicios de salud mental del Departamento de Salud 20 entre diciembre de 2012 y junio de 2013, y que desearon participar en el estudio voluntariamente. Como criterios de inclusión se exigió que los pacientes tenían que estar diagnosticados por su psiquiatra de al menos una de estas patologías: 1) Esquizofrenia; 2) Trastorno esquizofreniforme; 3) Trastorno psicoafectivo; 4) Otros trastornos de tipo psicótico; 5) Trastorno bipolar que en su tratamiento llevara de forma continuada medicación antipsicótica (American Psychiatric Association, 2000). Además, todos los pacientes tenían que estar en tratamiento con antipsicóticos atípicos (clozapina, olanzapina, risperidona, quetiapina, aripiprazol, ziprasidona, amisulprida, asenapina y paliperidona) durante al menos tres meses. Los pacientes que tomaban estos tipos de medicamentos fueron seleccionados porque el receptor HTR2C está involucrado en su mecanismo de acción, y este gen, a su vez, se asocia con el uso de sustancias psicoactivas (The Weizmann Institute of Science Crown Human Genome Centre, 2014).

\section{Variables y medidas}

La variable primaria del estudio fue la presencia del alelo C en el polimorfismo rs1414334 del gen HTR2C. A cada paciente seleccionado se le extrajo una muestra de sangre venosa (tubo de EDTA). Se aisló ADN genómico purificado de las muestras con capa leucocitaria, usando el 
sistema semiautomático QIAcube (Qiagen, Hilden, Alemania) según las instrucciones del fabricante. Se usó el ADN extraído para la genotipificación. El análisis del polimorfismo (rs1414334 C/G) se hizo mediante PCR en tiempo real para discriminación alélica (C_7455701_10, Applied Biosystems, Madrid, España) con aparatos TaqMan (Applied Biosystems 7300) (http://www.ncbi.nlm.nih.gov/projects/ SNP/snp_ref.cgi?rs=1414334). Las variables secundarias fueron: edad (años), sexo, esquizofrenia (sí/no), años desde el diagnóstico del trastorno, número de paquetes-año (número de cigarrillos al día $\mathrm{x}$ número de años fumando $\div 20$ ), criterios de síndrome metabólico (obesidad abdominal, hipertrigliceridemia, hipertensión, HDL-c anormal e insulinorresistencia) y SCORE (\%) (Conroy et al., 2003; Grundy et al., 2004). El diagnóstico principal y su duración, edad y sexo se obtuvieron de los historiales médicos de los pacientes. El número de cigarrillos y el tiempo que cada paciente había sido fumador fueron obtenidos mediante entrevista personal. Los criterios de síndrome metabólico se obtuvieron mediante medidas de presión arterial (sistólica y diastólica), analíticas de sangre (colesterol total, HDL-c, triglicéridos y glucemia en ayunas) y la circunferencia abdominal. Dichas medidas se obtuvieron siguiendo las guías clínicas vigentes (American Diabetes Association, 2012; National Institutes of Health, 1998; Mancia et al., 2007; National Cholesterol Education Program Expert Panel on Detection, Evaluation, and Treatment of High Blood Cholesterol in Adults, 2002). Finalmente, el SCORE se calculó usando la presión arterial sistólica, edad, sexo, índice aterogénico (colesterol total $\div$ HDL-c) y tabaco como variable binaria (fumador/no fumador).

\section{Tamaño de la muestra}

Dado que no se realizó un cálculo de tamaño de muestra a priori, calculamos la potencia estadística que ofrece la muestra recogida en el periodo del estudio: 166 pacientes, de los cuales 33 tenían el alelo $\mathrm{C}$ del polimorfismo estudiado. Con el objetivo de estimar un área bajo la curva ROC (ABC) diferente de 0,5, asumiendo un $\mathrm{ABC}$ de $0,70 \mathrm{y}$ un nivel de confianza del $95 \%$, se obtuvo una potencia del 94,43\% (Hanley y McNeil, 1982).

\section{Métodos estadísticos}

Se describieron las variables cualitativas mediante frecuencias absolutas y relativas, y las cuantitativas empleando medias y desviaciones estándar. Se calcularon las odds ratio (ORs) crudas para determinar posibles asociaciones de las variables secundarias con nuestra variable principal. Para obtener las ORs ajustadas, se construyó un modelo de regresión logística binaria por pasos, teniendo en cuenta que sólo podíamos introducir un máximo de 3 variables en el mismo (una por cada 10 eventos). Los pasos consistieron en obtener todas las combinaciones posibles de variables explicativas (231) y calcular el ABC del modelo resultante con dicha combinación, quedándonos con la combinación que tuviera una mayor ABC (modelo con mayor poder discriminatorio) (Azrak et al., 2015; Gutiérrez-Gómez et al., 2015; López- Bru, Palazón-Bru, Folgado-de la Rosa y Gil-Guillén, 2015; Ramírez-Prado et al., 2015). El error tipo I fue fijado en $5 \%$ y de cada parámetro relevante se calculó su intervalo de confianza asociado (IC). Los paquetes estadísticos empleados fueron el R 2.13.2. y el IBM SPSS Statistics 19 .

Tabla 1. Características descriptivas y analíticas de nuestra muestra de pacientes diagnosticados con trastorno psiquiátrico en una región de España: análisis del alelo C en el polimorfismo rs1414334 del gen HTR2C.

\begin{tabular}{|c|c|c|c|c|c|}
\hline Variable & $\begin{array}{r}\text { Total } n=166 \\
n(\%) / x \pm s\end{array}$ & $\begin{array}{r}\text { OR crudas } \\
\text { (IC 95\%) }\end{array}$ & p-valor & $\begin{array}{r}\text { OR ajustadas } \\
\text { (IC 95\%) }\end{array}$ & p-valor \\
\hline Edad (años) & $43,1 \pm 11,5$ & $1,02(0,98-1,05)$ & 0,318 & $\mathrm{~N} / \mathrm{M}$ & $\mathrm{N} / \mathrm{M}$ \\
\hline Sexo femenino & $67(40,4)$ & $2,40(1,11-5,22)$ & 0,027 & $4,31(1,67-11,17)$ & 0,003 \\
\hline Esquizofrenia & $92(55,4)$ & $0,52(0,24-1,12)$ & 0,094 & $\mathrm{~N} / \mathrm{M}$ & $\mathrm{N} / \mathrm{M}$ \\
\hline Años de diagnóstico del trastorno & $14,9 \pm 9,6$ & $0,99(0,95-1,03)$ & 0,486 & $\mathrm{~N} / \mathrm{M}$ & $\mathrm{N} / \mathrm{M}$ \\
\hline Número de paquetes-año & $11,6 \pm 17,5$ & $1,02(1,00-1,04)$ & 0,057 & $1,03(1,01-1,05)$ & 0,008 \\
\hline Obesidad abdominal* & $110(66,3)$ & $0,70(0,31-1,58)$ & 0,399 & $0,39(0,15-1,01)$ & 0,053 \\
\hline Hipertrigliceridemia* & $64(38,6)$ & $0,53(0,23-1,23)$ & 0,130 & $\mathrm{~N} / \mathrm{M}$ & $\mathrm{N} / \mathrm{M}$ \\
\hline Hipertensión arterial* & $58(34,9)$ & $0,76(0,33-1,73)$ & 0,511 & $N / M$ & $\mathrm{~N} / \mathrm{M}$ \\
\hline c-HDL alterado* & $55(33,1)$ & $0,85(0,37-1,94)$ & 0,698 & $N / M$ & $\mathrm{~N} / \mathrm{M}$ \\
\hline Resistencia a la insulina* & $31(18,7)$ & $0,54(0,18-1,67)$ & 0,261 & $\mathrm{~N} / \mathrm{M}$ & $\mathrm{N} / \mathrm{M}$ \\
\hline SCORE (\%) & $0,80 \pm 1,51$ & $1,20(0,96-1,50)$ & 0,107 & $\mathrm{~N} / \mathrm{M}$ & $N / M$ \\
\hline
\end{tabular}

Nota. $\mathrm{n}(\%)$, frecuencia absoluta (frecuencia relativa); x \pm s, media \pm desviación estándar; OR; odds ratio; IC, intervalo de confianza; N/M, no en el modelo. Bondad de ajuste del modelo multifactorial: X2 =14,80, $p=, 002$; área bajo la curva ROC =,706 (IC 95\%: 0,603- 0,810, p <,001); * Criterios para el Diagnóstico Clínico del Síndrome Metabólico (ATP III). 


\section{Resultados}

De un total de 166 pacientes tratados con antipsicóticos atípicos, 33 presentaron el alelo $\mathrm{C}$ del polimorfismo analizado (19,9\%, IC 95\%: 13,8-26,0\%). En la Tabla 1 observamos las características descriptivas y analíticas de nuestra muestra de pacientes. En ella destacamos una edad media de 43,1 años, un 55,4\% de esquizofrenia y una media de años con el diagnóstico principal de 14,9 años. Por otro lado, el consumo de tabaco se sitúo en 11,6 paquetes-año en media. Finalmente, en lo referente a los criterios de síndrome metabólico, éstos fueron muy prevalentes (obesidad abdominal, 66,3\%; hipertrigliceridemia, 38,6\%; hipertensión, 34,9\%; HDL-c anormal, 33,1\%; insulinorresistencia, $18,7 \%$ ).

Cuando valoramos factores asociados de forma univariante (Tabla 1) observamos una asociación estadísticamente significativa $(\mathrm{p}<0,05)$ del alelo $\mathrm{C}$ del polimorfismo analizado con ser mujer y mayor consumo tabáquico, mientras que quedo cerca de la significancia estadística $(0,05<\mathrm{p}<0,10)$ no tener esquizofrenia. Si realizamos un ajuste con la mejor combinación de factores $(\mathrm{ABC}=0,706)$, obtenemos que un mayor consumo tabáquico, ser mujer y no tener obesidad abdominal se asoció con el alelo analizado. El modelo que incluyó estos factores fue muy significativo $(p=0,002)$.

En la Figura 1 observamos las probabilidades pronosticadas de presencia del alelo $\mathrm{C}$ de nuestro polimorfismo. En ella se aprecia que dichas probabilidades aumentan conforme el paciente ha experimentado un consumo tabáquico mayor. Por otra parte, ser mujer y no tener obesidad abdominal mostraron mayor probabilidad de tener este alelo.

\section{Discusión}

\section{Resumen}

Este estudio determinó de forma innovadora la asociación entre el consumo tabáquico medido en paquetes-año y la presencia del alelo C del polimorfismo rs1414334 del gen HTR2C. Dicha asociación fue directa, es decir, a mayor consumo de tabaco por parte del paciente, mayor probabilidad de presentar este alelo. Por otra parte, se obtuvo que ser mujer y no tener obesidad abdominal se asoció con tener el alelo analizado.

\section{Fortalezas y limitaciones del estudio}

La fortaleza principal de este trabajo es la pregunta de investigación desarrollada, ya que no hemos encontrado trabajos que determinaron la asociación entre el tabaco y el alelo $\mathrm{C}$ del polimorfismo estudiado. Esto da carácter innovador a los resultados obtenidos. Por otra parte, se probaron todas las combinaciones posibles de variables para obtener el mejor modelo multivariante y la potencia de la muestra recogida fue cercana al $95 \%$, cuando la mayoría de estudios utilizan potencias de 80 y $90 \%$.
Figura 1. Las probabilidades previstas de la presencia del alelo $\mathrm{C}$ de nuestro polimorfismo rs1414334 del gen HTR2C según variables específicos en el modelo multifactorial.

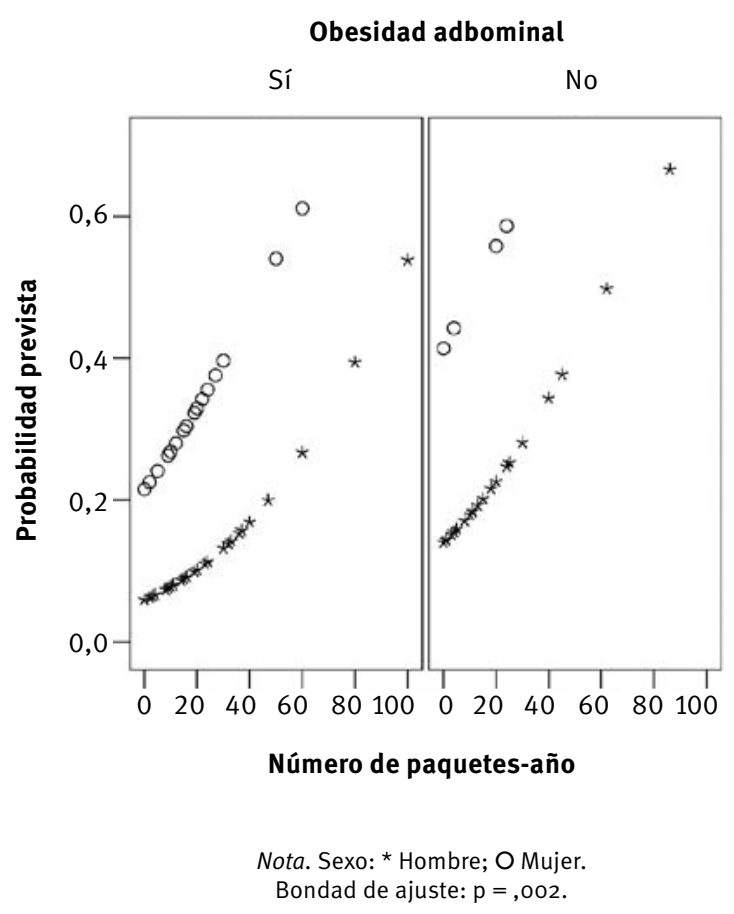

Para minimizar el sesgo de selección, se recogieron datos de pacientes en un periodo de tiempo determinado. Respecto al sesgo de información, los datos fueron recogidos de forma rigurosa y exhaustiva por el equipo investigador. Finalmente, el sesgo de confusión fue minimizado utilizando la mejor combinación de un total de 231.

Nuestros hallazgos es probable que estén relacionados con la naturaleza del gen HTR2C que se encuentra ligado al cromosoma $\mathrm{X}$ (es decir, los varones son hemicigotos) perdiendo poder estadístico. Además, algunos antipsicóticos de segunda generación, como asenapina, clozapina, olanzapina y sertindol, son antagonistas relativamente potentes de los receptores 5-HT, y otros antipsicóticos como amisulprida, asenapina, clozapina, lurasidona y risperidona, tienen una elevada afinidad por los receptores 5-HT. Los efectos de estos antipsicóticos de segunda generación pueden distorsionar una posible relación con las variaciones genéticas en la actividad del receptor.

Por lo tanto, la relación entre el polimorfismo 5-HT2C y el tabaquismo puede deberse al camuflaje debido a la unión al receptor causada por el tratamiento farmacológico actual. Por otro lado, el tabaquismo se midió mediante una entrevista clínica, en lugar de con el CO espirado.

Comparación con la literatura existente

A la hora de valorar la asociación entre el consumo de tabaco y el alelo estudiado, no hemos encontrado trabajos para comparar nuestros resultados, ya que los estudios previos no consideraron el tabaco entre sus variables (Gregoor et al., 2010; Hoekstra et al., 2010; Houston et al., 2012; Kle- 
mettilä et al., 2015; Ma et al., 2014; Mulder et al., 2007a; Mulder et al., 2007b; Mulder et al., 2009; Rico-Gomis et al., 2016; Risselada et al., 2012). Nosotros pensamos que esta asociación puede estar en relación con el papel del receptor HTR2C en la modulación de la nicotina a nivel cerebral, ya que un comportamiento similar se ha apreciado en modelos animales con ratones (Fletcher et al., 2008; Guy y Fletcher, 2014). No obstante, esto debería de corroborarse con estudios genéticos y experimentales.

Con respecto al resto de asociaciones encontradas, el sexo es concordante, ya que este gen se encuentra localizado en el cromosoma X. Finalmente, la asociación de la obesidad abdominal con este alelo no queda clara en los dos estudios anteriores publicados sobre el tema, ya que uno de ellos encontró una asociación directa y el otro no encontró asociación entre estas dos variables (OR cercana a 1) (Mulder et al., 2007a; Risselada et al., 2012).

El alelo C se presentó aproximadamente en uno de cada cinco pacientes tratados con antipsicóticos atípicos, los cual es similar a los resultados encontrados por otros autores (Hoekstra et al., 2010; Mulder et al., 2007a; Mulder et al., 2007b; Mulder et al., 2009; Risselada et al., 2012).

\section{Implicaciones para la investigación y la práctica}

Dado que el consumo de tabaco se ha asociado al alelo C del polimorfismo rs1414334 del gen HTR2C, si estos resultados se confirman con otros estudios, podremos determinar qué pacientes tienen mayor probabilidad de consumo elevado de tabaco y sobre ellos realizar de forma precoz, intervenciones para conseguir que el paciente disminuya parcialmente o totalmente su consumo de tabaco y esto repercutirá en una disminución del riesgo cardiovascular (Prescott, Hippe, Schnohr, Hein, y Vestbo,1998), el cual es más elevado que en la población general (McEvoy et al., 2005; Newcomer y Hennekens, 2007; Saha, Chant y McGrath, 2007).

Según los resultados obtenidos, se podría realizar un estudio analizando el alelo $\mathrm{C}$ en una muestra de mayor tamaño, comparando la población fumadora con la no fumadora, incluyendo a varones y mujeres, y estratificando por historial psiquiátrico y síndrome metabólico. Además, se podrían incluir variables relacionadas con el tabaquismo, tales como los intentos de abandono, el éxito/ fracaso de la deshabituación, y los fármacos usados para el cese tabáquico.

\section{Conclusiones}

Este estudio ha encontrado que existe asociación directa entre un mayor consumo de tabaco a lo largo de los años y la presencia del alelo C del polimorfismo rs1414334 del gen HTR2C. Dado que no hemos encontrado estudios que valoraran dicha asociación, se necesitan trabajos que co- rroboren nuestros resultados. En caso de verificarse dicha asociación, conoceremos qué pacientes tratados con antipsicóticos atípicos tienen mayor probabilidad de un consumo tabáquico elevado.

\section{Reconocimientos}

Los autores desean expresar su agradecimiento a Maria Repice e Ian Johnstone por sus servicios de traducción y de revisión de la versión final de este manuscrito.

\section{Conflicto de intereses}

Los autores declaran la inexistencia de conflicto de intereses.

\section{Referencias}

American Diabetes Association. (2012). Standards of medical care in diabetes-2012. Diabetes Care, 35, S11-63. doi:10.2337/dc12-s011.

American Psychiatric Association. (2000). Diagnostic and Statistical Manual of Mental Disorders. 4th edition. Washington: American Psychiatric Press.

Azrak, C., Palazón-Bru, A., Baeza-Díaz, M. V., Folgado-De la Rosa, D. M., Hernández-Martínez, C., Martínez-Toldos, J. J. y Gil-Guillén, V. F. (2015). A predictive screening tool to detect diabetic retinopathy or macular edema in primary health care: construction, validation and implementation on a mobile application. PeerJ, 3, e1404. doi:10.7717/peerj.1404.

Barrot, C., Sánchez, C., Abellana, R., Ortega, M. y Gené, M. (2013). Polimorfismos genéticos como indicadores de la vulnerabilidad individual a la adicción al tabaco. Medicina Clínica (Barcelona), 140, 49-52. doi:10.1016/j. medcli.2012.05.041.

Conroy, R. M., Pyörälä, K., Fitzgerald, A. P., Sans, S., Menotti, A., De Backer, G., Graham, I. M.; SCORE project group. (2003). Estimation of ten-year risk of fatal cardiovascular disease in Europe: the SCORE project. European Heart Journal, 24, 987-1003. doi:10.1016/ S0195668X(03)00114-3.

Fletcher, P. J., Lê, A. D. y Higgins, G. A. (2008). Serotonin receptors as potential targets for modulation of nicotine use and dependence. Progress in Brain Research, 172, 361383. doi:10.1016/S0079-6123(08)00918-7.

Gregoor, J. G., Mulder, H., Cohen, D., van Megen, H. J., Egberts, T. C., Heerdink, E. R. y van der Weide, J. (2010). Combined HTR2C-LEP genotype as a determinant of obesity in patients using antipsychotic medication. Journal of Clinical Psychopharmacology, 30, 702-705. doi:10.1097/JCP.0b013e3181fa05a2.

Grundy, S. M., Brewer, H. B. Jr, Cleeman, J. I., Smith, S. C. Jr y Lenfant, C; American Heart Association; Na- 
tional Heart, Lung, and Blood Institute. (2004). Definition of metabolic syndrome: Report of the National Heart, Lung, and Blood Institute/American Heart Association conference on scientific issues related to definition. Circulation, 109, 433-438. doi:10.1161/01. CIR.0000111245.75752.C6.

Guy, E. G. y Fletcher, P. J. (2014). Responding for a conditioned reinforcer, and its enhancement by nicotine, is blocked by dopamine receptor antagonists and a 5 -HT (2C) receptor agonist but not by a 5-HT(2A) receptor antagonist. Pharmacology Biochemistry and Behavior, 125, 40-47. doi:10.1016/j.pbb.2014.08.006.

Gutiérrez-Gómez, T., Cortés, E., Palazón-Bru, A., Peñarrieta- de Córdova, I., Gil-Guillén, V. F. y Ferrer-Diego, R. M. (2015). Six simple questions to detect malnutrition or malnutrition risk in elderly women. PeerJ, 3, e1316. doi:10.7717/peerj.1316.

Hanley, J. A. y McNeil, B. J. (1982). The meaning and use of the area under a receiver operating characteristic (ROC) curve. Radiology, 143, 29-36. doi:10.1148/radiology. 143.1.7063747.

Hoekstra, P. J., Troost, P. W., Lahuis, B. E., Mulder, H., Mulder, E. J., Franke, B., Buitelaar, J. K., Anderson, G. M., Scahill, L. y Minderaa, R. B. (2010). Risperidone-induced weight gain in referred children with autism spectrum disorders is associated with a common polymorphism in the 5-hydroxytryptamine 2C receptor gene. Journal of Child and Adolescent Psychopharmacology, 20, 473-477. doi:10.1089/cap.2009.0071.

Houston, J. P., Kohler, J., Bishop, J. R., Ellingrod, V. L., Ostbye, K. M., Zhao, F., Conley, R. R., Poole Hoffmann, V. y Fijal, B. A. (2012). Pharmacogenomic associations with weight gain in olanzapine treatment of patients without schizophrenia. Journal of Clinical Psychiatry, 73, 1077-1086. doi:10.4088/JCP.11m06916.

Klemettilä, J. P., Kampman, O., Seppälä, N., Viikki, M., Hämäläinen, M., Moilanen, E., Mononen, N., Lehtimäki, T. y Leinonen, E. (2015). Association study of the HTR2C, leptin and adiponectin genes and serum marker analyses in clozapine treated long-term patients with schizophrenia. European Psychiatry, 30, 296-302. doi:10.1016/j.eurpsy.2014.08.006.

López-Bru, D., Palazón-Bru, A., Folgado-de la Rosa, D. M. y Gil-Guillén, V. F. (2015). Scoring System for Mortality in Patients Diagnosed with and Treated Surgically for Differentiated Thyroid Carcinoma with a 20-Year Follow- Up. PLoS One, 10, e0128620. doi:10.1371/journal. pone.0128620.

Ma, X., Maimaitirexiati, T., Zhang, R., Gui, X., Zhang, W., $\mathrm{Xu}, \mathrm{G}$. y Hu, G. (2014). HTR2C polymorphisms, olanzapine- induced weight gain and antipsychotic-induced metabolic syndrome in schizophrenia patients: a metaanalysis. International Journal of Psychiatry in Clinical Practice, 18, 229-242. doi:10.3109/13651501.2014.957705.
Mancia, G., De Backer, G., Dominiczak, A., Cifkova, R., Fagard, R., Germano, G., Williams, B. (2007). 2007 Guidelines for the Management of Arterial Hypertension: The Task Force for the Management of Arterial Hypertension of the European Society of Hypertension (ESH) and of the European Society of Cardiology (ESC). Journal of Hypertension, 25, 1105-1187. doi:10.1097/HJH. 0b013e3281fc975a.

McEvoy, J. P., Meyer, J. M., Goff, D. C., Nasrallah, H. A., Davis, S. M., Sullivan, L., Lieberman, J. A. (2005). Prevalence of the metabolic syndrome in patients with schizophrenia: baseline results from the Clinical Antipsychotic Trials of Intervention Effectiveness (CATIE) schizophrenia trial and comparison with national estimates from NHANES III. Schizophrenia Research, 80, 1932. doi:10.1016/j.schres.2005.07.014.

Mulder, H., Franke, B., van der-Beek van der, A. A., Arends, J., Wilmink, F. W., Scheffer, H. y Egberts, A. C. (2007). The association between HTR2C gene polymorphis$\mathrm{ms}$ and the metabolic syndrome in patients with schizophrenia. Journal of Clinical Psychopharmacology, 27, 338343. doi:10.1097/JCP.0b013e3180a76dc0.

Mulder, H., Franke, B., van der-Beek van der, A. A., Arends, J., Wilmink, F. W., Egberts, A. C. y Scheffer, H. (2007). The association between HTR2C polymorphisms and obesity in psychiatric patients using antipsychotics: a cross-sectional study. Pharmacogenomics Journal, 7, 318324. doi:10.1038/sj.tpj.6500422.

Mulder, H., Cohen, D., Scheffer, H., Gispen-de Wied, C., Arends, J., Wilmink, F. W., Egberts, A. C. (2009). HTR2C gene polymorphisms and the metabolic syndrome in patients with schizophrenia: a replication study. Journal of Clinical Psychopharmacology, 29, 16-20. doi:10.1097/JCP. 0b013e3181934462.

National Cholesterol Education Program (NCEP) Expert Panel on Detection, Evaluation, and Treatment of High Blood Cholesterol in Adults (Adult Treatment Panel III). (2002). Third Report of the National Cholesterol Education Program (NCEP) Expert Panel on Detection, Evaluation, and Treatment of High Blood Cholesterol in Adults (Adult Treatment Panel III) final report. Circulation, 106, 3143-3421.

National Institutes of Health. (1998). Clinical Guidelines on the Identification, Evaluation, and Treatment of Overweight and Obesity in Adults-The Evidence Report. Obesity Research, 6, 51S-209S. doi:10.1002/j.1550-8528.1998. tb00690.x.

Newcomer, J. W. y Hennekens, C. H. (2007). Severe mental illness and risk of cardiovascular disease. JAMA, 298, 1794-1796. doi:10.1001/jama.298.15.1794.

Prescott, E., Hippe, M., Schnohr, P., Hein, H. O. y Vestbo, J. (1998). Smoking and risk of myocardial infarction in women and men: longitudinal population study. BMJ, 316, 1043-1047. doi:10.1136/bmj.316.7137.1043. 
Ramírez-Prado, D., Palazón-Bru, A., Folgado-de la Rosa, D. M., Carbonell-Torregrosa, M. Á., Martínez-Díaz, A. M., Martínez-St John, D. R. y Gil-Guillén, V. F. (2015). A four-year cardiovascular risk score for type 2 diabetic inpatients. PeerJ, 3, e984. doi:10.7717/peerj.984.

Rico-Gomis, J. M., Palazón-Bru, A., Triano-García, I., Mahecha- García, L. F., García-Monsalve, A., Navarro-Ruiz, A., ...Gil-Guillén, V. F. (2016). Association between the HTR2C rs1414334 C/G gene polymorphism and the development of the metabolic syndrome in patients treated with atypical antipsychotics. PeerJ, 4, e2163. doi:10.7717/peerj.2163.

Risselada, A. J., Vehof, J., Bruggeman, R., Wilffert, B., Cohen, D., Al Hadithy, A. F., ... Mulder, H. (2012). Association between HTR2C gene polymorphisms and the metabolic syndrome in patients using antipsychotics: a replication study. Pharmacogenomics Journal, 12, 62- 67. doi:10.1038/tpj.2010.66.

Saccone, S. F., Hinrichs, A. L., Saccone, N. L., Chase, G. A., Konvicka, K., Madden, P. A., ... Bierut, L. J. (2007). Cholinergic nicotinic receptor genes implicated in a nicotine dependence association study targeting 348 candidate genes with 3713 SNPs. Human Molecular Genetics, 16, 36-49. doi:10.1093/hmg/ddl438.

Saha, S., Chant, D. y McGrath, J. (2007). A systematic review of mortality in schizophrenia: is the differential mortality gap worsening over time? Archives of General Psychiatry, 64, 1123-1131. doi:10.1001/archpsyc.64.10.1123.

San, L., Arranz, B., Arrojo, M., Becoña, E., Bernardo, M., Caballero, L., ...Zorrilla, I. (2016). Clinical guideline for the treatment of dual pathology in the adult population. Adicciones, 28, 3-5. doi:10.20882/adicciones.784.

The Weizmann Institute of Science Crown Human Genome Centre. (2014). GeneCards. Retrieved at http:/ / www. genecards.org/cgi bin/carddisp.pl?gene=HTR2C.

Verde, Z., Santiago, C., Rodríguez González-Moro, J. M., de Lucas Ramos, P., López Martín, S., Bandrés, F., ... Gómez-Gallego, F. (2011). 'Smoking genes': a genetic association study. PLoS One, 6, e26668. doi:10.1371/ journal. pone.0026668.

Walton, R., Johnstone, E., Munafò, M., Neville, M. y Griffiths, S. (2001). Genetic clues to the molecular basis of tobacco addiction and progress towards personalized therapy. Trends in Molecular Medicine, 7, 70-76. doi:10.1016/ S1471-4914(01)01915-3.

Wilson, P. W., D’Agostino, R. B., Levy, D., Belanger, A. M., Silbershatz, H. y Kannel, W. B. (1998). Prediction of coronary heart disease using risk factor categories. Circulation, 97, 1837-1847. doi:10.1161/01.CIR.97.18.1837. 